\title{
Reconsidering Antimalarials in Systemic Lupus Erythematosus: Developments of Translational Clinical Interest
}

Successful trials of biologic therapies in systemic lupus erythematosus (SLE) have raised interest in targeting a variety of additional putative mechanisms of action. These include preparations that antagonize Toll-like receptors (TLR) 7 and 9 , one of the actions of antimalarials. Although they have been studied for over a hundred years, the last decade has witnessed a flurry of reports demonstrating novel physiologic effects of these agents. Prospective and retrospective studies that document disease-modifying outcomes of antimalarials have appeared. This report summarizes those breakthroughs and insights, which are important in our approach toward SLE management.

Historical context. According to legend, bark from a "fever tree" in 1620s Peru miraculously cured the mysterious illness of the Countess of Cinchona. That event led the Jesuits in Europe to form the first modern cartel - one that controlled the international production and distribution of quinine by the $1660 \mathrm{~s}^{1}$. The first modern clinical investigations of quinine for rheumatic disorders were not published until Payne's report of its efficacy for cutaneous lupus in $1894^{2}$ and were expanded upon in the 1920s and 1930s. The quinacrine story is among the most colorful in the annals of pharmacologic interventions. It was the last discovery by German bacteriologist Paul Ehrlich before he died in 1915. In the United States, President Franklin D. Roosevelt's administration and congressional leadership had to go to court to get pro-German sympathizers at the Winthrop Chemical Company to disclose the quinacrine manufacturing process. It was used by over 3 million Allied soldiers daily during World War II for protection from malaria. There are even literary references to quinacrine, ranging from Joseph Heller in Catch-22 to Rodgers and Hammerstein's musical South Pacific ${ }^{3,4,5}$. Evidence-based literature published as early as 1951 and through the 1960s documented that quinacrine, chloroquine (CQ), and hydroxychloroquine (HCQ) improved rheumatic disorders. By 1970, diverse mechanisms of quinacrine action (e.g., antineoplastic, antiinflammatory, antimicrobial, antiarrhythmic) were already well described ${ }^{6}$.

Lysosomotropy and cellular pH: initial insights. Until the last decade, the immunomodulatory effects of antimalarials have been largely attributed to their lysosomotropic action. Antimalarials are weak basic compounds that show affinity for the acidic endoplasm of lysosomes and alter the $\mathrm{pH}$ of endolysosomal compartments. Seminal work by Robert Fox and his colleagues at the Scripps Clinic in La Jolla, California, demonstrated how HCQ impaired the process of lysosomal acidification, which is vital for lysosome function ${ }^{7}$. Lysosomes perform a dual function in T cells, including degrading the endocytosed cellular matter as well as participating in the apoptosis of antigen-presenting cells. Thus, impaired lysosome function results in dysregulation of immune responses and also causes further downstream effects including cytokine inhibition. Our group also showed that HCQ exerts a long-lasting suppressive effect on interleukin 6 levels, possibly by affecting macrophage/ monocyte-released as opposed to lymphocyte-released cytokines $^{8,9}$.

TLR7 and TLR9 and newer lysosomotropy insights. More recently, the discovery of TLR has led to a paradigm shift in our understanding of the role of the innate immune system in SLE. TLR are unique components of innate immunity that work by pathogen-associated molecular pattern (PAMP) recognition. These receptors recognize the molecular pattern of antigens and activate the innate immune system in case of pathogenic invasion. This mechanism does not confer TLR with a high degree of specificity but makes them an extremely important first line of defense in recognition of non-self nucleic material. However, when the regulatory process for PAMP breaks down, TLR cannot differentiate self-nucleic acids from non-self antigens and erroneously activate the immune response.

In SLE, the immune complexes formed by DNA and RNA form ligands for TLR activation. The effects of TLR7 (interacts with ssRNA ligand) and TLR9 (interacts with dsDNA, e.g., CpG-DNA ligands) have been studied in great detail, and recent data have shown that antimalarials inhibit lysosomal TLR function ${ }^{10}$. The exact mechanism of this influence is not known. Nucleic acid-sensing TLR are located intracellularly to minimize their potential reactivity against nucleic acids on the cell surface, and they are activated only when the foreign nucleic acids are presented to them by intermediate molecules such as Fc- $\gamma$ receptor. These intracellular TLR typically undergo processing by endosomes before becoming active, and it has been proposed that antimalarials inhibit the endosomal acidification and thus inhibit the TLR activation.

Recently, Kuznik, et al demonstrated in vitro that this Personal non-commercial use only. The Journal of Rheumatology Copyright $@$ () 2012. All rights reserved. 
inhibitory effect may involve mechanisms independent of endosomal acidification ${ }^{11}$. In their investigation, antimalarials mechanically inhibited nucleic acids binding to TLR. They proposed that TLR-binding epitopes of nucleic acids were masked by antimalarials molecules, thus blocking the possible interaction between TLR and nucleic material (Figure 1).

Antimalarials, antagonists to TLR7 and TLR9, and SLE. In 2007 , the first head-to-head studies ${ }^{12}$ demonstrated that in animal models of SLE, chloroquine had actions similar to those of endosomal TLR antagonists (Figure 2).

Although that particular compound is no longer being developed, several other companies are working on early-phase studies with TLR7 or TLR9 antagonists. These companies include ESAI (Worcester, MA, USA), Dynavax (Berkeley, CA, USA; DV 1179, GlaxoSmithKline), and Idera (IMO 1300; Cambridge, MA, USA). P13 peptide is also being studied (13therapeutics ${ }^{\mathrm{TM}}$, Oregon Health and Science University, Portland, OR, USA). Most of these antagonists are orally or subcutaneously administered small molecules ${ }^{13}$. A kinder, gentler HCQ for SLE? An exciting opportunity to prepare "improved" formations of HCQ lies in its enantiomeric metabolism. Both CQ and HCQ undergo stereospecific metabolism, resulting in formation of $\mathrm{R}$ - and
S-enantiomers. Data have suggested that the S-enantiomer has a shorter half-life and lower blood levels because of its preferential metabolism ${ }^{14}$. As an extension of this finding, it has been suggested that more targetted formulations of HCQ containing S-enantiomer may reduce the risk of retinopathy, considering HCQ's affinity for pigmented tissues and its enantiospecific tissue disposition ${ }^{15}$.

A new sense of urgency and relevance. As case reports give way to case series and retrospective surveys to prospective cohorts, many of the perceptions regarding antimalarials in SLE are now on even firmer ground. It appears that patients taking HCQ, for example, have less damage accrual, prolonged survival, and improved renal and skin outcomes. Additionally, HCQ delays the onset of SLE in those at risk, lowers damage scores, causes less vascular damage, leads to fewer infections and thromboembolic events, decreases lipid levels, decreases congenital heart block prevalence, and ameliorates inflammatory arthritis and Sjögren's manifestations $16,17,18,19,20$. The pharmaceutical industry now has 2 options: to decrease the toxicity of HCQ by developing a purer formulation, or to develop an HCQ-like TLR antagonist. Let us hope that both options are explored. There is too much at stake for millions of patients with SLE throughout the world.

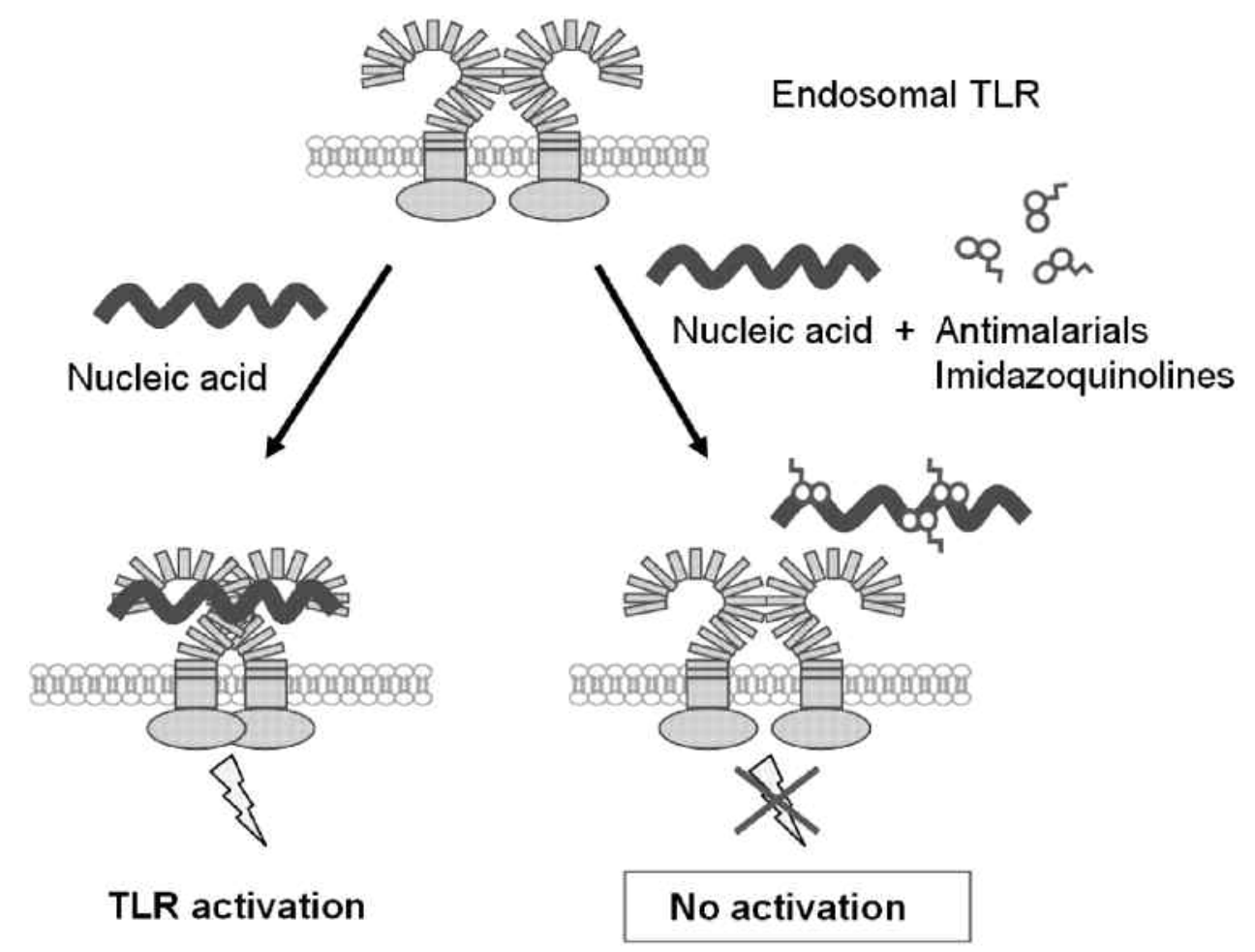

Figure 1. Antimalarials molecules mask Toll-like receptor (TLR)-binding epitopes of nucleic acids, blocking the possible interaction between TLR and nucleic material ${ }^{11}$. From Kuznik, et al. J Immunol 2011;186:4794-804; with permission. 


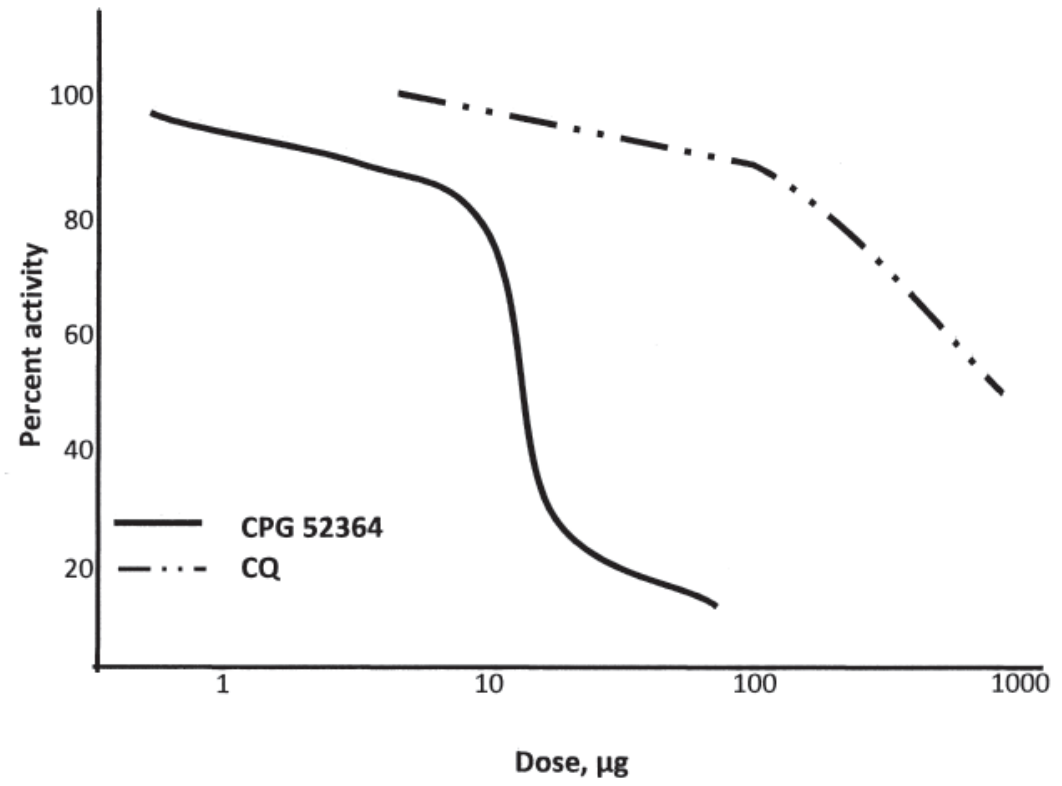

Figure 2. CpG 52364 showed dose-dependent inhibition of Toll-like receptor 9-mediated intraperitoneal (IP)-10 induction in mice. Female adult BALB/c mice received different doses of antagonist compounds by IP injection or oral gavage. At $1 \mathrm{~h}$ post-IP dose or $4 \mathrm{~h}$ post-oral dose, animals received $100 \mu \mathrm{g}$ CpG-DNA oligodeoxynucleotide subcutaneously. Plasma collected at $3 \mathrm{~h}$ post-agonist injection was used for IP-10 assay by ELISA. Data are reported as the $50 \%$ inhibitory dose. CQ: chloroquine. Figure courtesy A.M. Krieg.

\section{SWAMY VENUTURUPALLI, MD, FACR; VINEET GUDSOORKAR, MBBS; DANIEL WALLACE, MD, FACR,} Cedars Sinai Medical Center - Rheumatology, 8737 Beverly Blvd., Suite 203,

Los Angeles, California 90048, USA

Address correspondence to Dr. Venuturupalli;

E-mail: swamy.venuturupalli@gmail.com

\section{REFERENCES}

1. Wallace DJ. The history of antimalarials. Lupus 1996;5 Suppl 1:S2-3.

2. Payne JF. A post-graduate lecture on lupus erythematosus. Clin J $1894 ; 4: 223$.

3. Ambruster HW. Treason's peace, German dyes and American dupes. New York: Beechhurst Press; 1947.

4. Heller J. Catch-22. New York: Simon \& Shuster; 1955:106.

5. Rodgers R, Hammerstein O. South Pacific. 1949.

6. Mackenzie AH. An appraisal of chloroquine. Arthritis Rheum 1970;13:280-91.

7. Fox RI, Kang HI. Mechanism of action of antimalarial drugs: Inhibition of antigen processing and presentation. Lupus 1993;2 Suppl 1:S9-12.

8. Wallace DJ, Linker-Israeli M, Hyun S, Klinenberg JR, Stecher V. The effect of hydroxychloroquine therapy on serum levels of immunoregulatory molecules in patients with systemic lupus erythematosus. J Rheumatol 1994;21:375-6.

9. Jang C, Choi J, Byun M, Jue D. Chloroquine inhibits production of TNF- $\alpha$, IL- $1 \beta$ and IL- 6 from lipopolysaccharide-stimulated human monocytes/macrophages by different modes. Rheumatology 2006;45:703-10.

10. Lafyatis R, York M, Marshak-Rothstein A. Antimalarial agents: Closing the gate on toll-like receptors? Arthritis Rheum 2006;54:3068-70.

11. Kuznik A, Bencina M, Svajger U, Jeras M, Rozman B, Jerala R. Mechanism of endosomal TLR inhibition by antimalarial drugs and imidazoquinolines. J Immunol 2011;186:4794-804.
12. Lipford G, Forsbach A, Zepp C, Nguyen T, Weeratna R, McCluskie $\mathrm{M}$, et al. Selective Toll-like receptor 7/8/9 antagonists for the oral treatment of autoimmune diseases. Arthritis Rheum 2007;56 Suppl:S628. [Internet. Accessed April 12, 2012.] Available from: http://acr.confex.com/acr/2007/webprogram/Paper8044.html

13. Wallace DJ. Advances in drug therapy for systemic lupus erythematosus. BMC Medicine 2010;8:77.

14. Tett SE, McLachlan AJ, Cutler DJ, Day RO. Pharmacokinetics and pharmacodynamics of hydroxychloroquine enantiomers in patients with rheumatoid arthritis receiving multiple doses of racemate. Chirality 1994;6:355-9.

15. Wallace DJ. Antimalarials - The 'real' advance in lupus. Lupus 2001;10:385-7.

16. Fessler BJ, Alarcón GS, McGwin G, Roseman J, Bastian HM, Friedman AW, et al, and for the LUMINA Study Group. Systemic lupus erythematosus in three ethnic groups: XVI. Association of hydroxychloroquine use with reduced risk of damage accrual. Arthritis Rheum 2005;52:1473-80.

17. Molad Y, Gorshtein A, Wysenbeek AJ, Guedj D, Majadla R, Weinberger A, et al. Protective effect of hydroxychloroquine in systemic lupus erythematosus. Prospective long-term study of an Israeli cohort. Lupus 2002;11:356-61.

18. Izmirly PM, Kim MY, Llanos C, Le PU, Guerra MM, Askanase AD, et al. Evaluation of the risk of anti-SSA/Ro-SSB/La antibody-associated cardiac manifestations of neonatal lupus in fetuses of mothers with systemic lupus erythematosus exposed to hydroxychloroquine. Ann Rheum Dis 2010;69:1827-30.

19. Becker-Merok A, Nossent J. Prevalence, predictors and outcome of vascular damage in systemic lupus erythematosus. Lupus 2009;18:508-15.

20. Kasitanon N, Fine DM, Haas M, Magder LS, Petri M. Hydroxychloroquine use predicts complete renal remission within 12 months among patients treated with mycophenolate mofetil therapy for membranous lupus nephritis. Lupus 2006;15:366-70.

J Rheumatol 2012;39:1769-71; doi:10.3899/jrheum.111616 